\title{
PERMUTATION REPRESENTATION OF GROUPS WITH BOOLEAN ORTHOGONALITIES
}

\author{
GARY DAVIS \\ (Received 20 June 1977; revised 1 October 1979) \\ Communicated by E. Strzelecki
}

\begin{abstract}
Reduced rings and lattice-ordered groups are examples of groups with Boolean orthogonalities. In this note we show that any group with a Boolean orthogonality satisfying a finiteness condition introduced by Stewart is isomorphic with a group of homeomorphisms of a topological space, in which two homeomorphisms are orthogonal if and only if they have disjoint supports.
\end{abstract}

1980 Mathematics subject classification (Amer. Math. Soc.): primary 54 H 10; secondary 06 F 15, $54 \mathrm{H} 15,57 \mathrm{~S} 99$.

\section{Introduction}

Groups with Boolean orthogonalities were introduced by Davis (1971a) principally to obtain representation and extension theorems for lattice-ordered groups and reduced rings in a uniform way. Since then Boolean orthogonalities on groups and rings have been studied by Davis (1971b), (1975) and Stewart (1975). A universal algebraic study of algebras with Boolean orthogonalities has been undertaken by Cornish (1975) and Cornish and Stewart (1977).

In this note we show that every group $G$ with a Boolean orthogonality $\perp$ satisfying a certain finiteness condition can be faithfully represented as a group of permutations $\hat{G}=\{\hat{g}: g \in G\}$ of a set $X$ in such a way that $g \perp h$ holds if and only if $\{x \in X: x \hat{g}=x\} \cup\{x \in X: x \hat{h}=x\}=X$, for all $g h \in G$.

C Copyright Australian Mathematical Society 1981 


\section{Boolean orthogonalities on permutation groups}

Let $G$ denote a group and $\perp$ a binary relation on $G$. For a non-empty subset $S$ of $G$ we define $S^{\perp}=\{g \in G: g \perp h$ for all $h \in S\}$ and $S^{\perp \perp}=\left(S^{\perp}\right)^{\perp}$. If $S=\{h\}$ is a singleton set then we denote $S^{\perp}$ and $S^{\perp \perp}$ by $h^{\perp}$ and $h^{\perp \perp}$ respectively.

The relation $\perp$ is a Boolean orthogonality on $G$ if for each non-empty subset $S$ of $G$ the set $S^{\perp}$ is a subgroup of $G$ and, in addition,

(1) $\perp$ is a symmetric relation,

(2) if $g \perp g$ then $g=1$,

(3) if $g \perp h$ then $k^{-1} g k \perp k^{-1} h k$ for all $k \in G$,

(4) if $g^{\perp \perp} \cap h^{\perp \perp}=1$ then $g \perp h$.

THEOREM 1 (Davis (197lb)). Let $G$ be a group with a Boolean orthogonality $\perp$. Then $B(G)=\left\{S^{\perp}: S\right.$ is a non-empty subset of $\left.G\right\}$, ordered by inclusion, is a complete Boolean algebra with $A \wedge B=A \cap B$ and $A \vee B=\left(A^{\perp} \cap B^{\perp}\right)^{\perp}$ for all $A, B \in B(G)$.

Let $G$ be a subgroup of the symmetric group on a set $X$. The support of $g \in G$ is the set $\operatorname{supp}(g)=\{x \in X: g h \neq x\}$. We shall say that $G$ is a $d$-group (for "directed group") if whenever $g_{1}, g_{2} \in G$ and $\varnothing \neq \operatorname{supp}\left(g_{1}\right) \cap \operatorname{supp}\left(g_{2}\right)$ there is an $h \in G$ with $\varnothing \neq \operatorname{supp}(h) \subseteq \operatorname{supp}\left(g_{1}\right) \cap \operatorname{supp}\left(g_{2}\right)$.

Proposition 2. Let $G$ be a subgroup of the symmetric group on $X$. Define the binary relation $\perp$ on $G$ by $g \perp h$ if $\operatorname{supp}(g) \cap \operatorname{supp}(h) \neq \varnothing$. If $G$ is a d-group then $\perp$ is a Boolean orthogonality on $G$. Conversely if $\perp$ is a Boolean orthogonality and for each $(x, g) \in X \times G$ with $x g=x$ there is an $h \in G$ with $x \in \operatorname{supp}(h)$ and $\operatorname{supp}(g) \cap \operatorname{supp}(h)=\varnothing$, then $G$ is a d-group.

Proof. Suppose $G$ is a $d$-group. The relation $\perp$ is clearly symmetric and satisfies (2) of the definition of a Boolean orthogonality. Furthermore each $S^{\perp}$ is clearly a subgroup of $G$ and condition (3) follows from the fact that $\operatorname{supp}\left(k^{-1} g k\right)=\operatorname{supp}(g) k$ for all $g, k \in G$. Suppose that $g, h \in G$ and $g \perp h$ does not hold. Then $\operatorname{supp}(g) \cap \operatorname{supp}(h) \neq \varnothing$ so there is a $k \in G$ with $\varnothing \neq$ $\operatorname{supp}(k) \subseteq \operatorname{supp}(g) \cap \operatorname{supp}(h)$. If $g^{\prime} \in g^{\perp}$ then $\operatorname{supp}\left(g^{\prime}\right) \cap \operatorname{supp}(k) \subseteq \operatorname{supp}\left(g^{\prime}\right)$ $\cap \operatorname{supp}(g)=\varnothing$ so $g^{\prime} \in k^{\perp}$. That is $g^{\perp} \subseteq k^{\perp}$. Similarly $h^{\perp} \subseteq k^{\perp}$ so $1 \neq k \in$ $g^{\perp \perp} \cap h^{\perp \perp}$, and therefore $\perp$ is a Boolean orthogonality.

Suppose conversely that $\perp$ is a Boolean orthogonality and for all $(x, g) \in X$ $\times G$ with $x g=x$ there is a $g^{\prime} \in G$ with $x \in \operatorname{supp}\left(g^{\prime}\right)$ and $\operatorname{supp}(g) \cap \operatorname{supp}\left(g^{\prime}\right)$ $=\varnothing$. Assume that $g, h \in G$ and $\operatorname{supp}(g) \cap \operatorname{supp}(h) \neq \varnothing$. Since $\perp$ is a Boolean orthogonality we can find $1 \neq k \in g^{\perp \perp} \cap h^{\perp \perp}$. 
Suppose $x \notin \operatorname{supp}(g)$. Then there is a $g^{\prime} \in G$ with $x \in \operatorname{supp}\left(g^{\prime}\right)$ and $\operatorname{supp}(g)$ $\cap \operatorname{supp}\left(g^{\prime}\right)=\varnothing$, so $\operatorname{supp}\left(g^{\prime}\right) \cap \operatorname{supp}(k)=\varnothing$ and therefore $x \notin \operatorname{supp}(k)$. Similarly $x \notin \operatorname{supp}(h)$ implies $x \notin \operatorname{supp}(k)$ so $\varnothing \neq \operatorname{supp}(k) \subseteq \operatorname{supp}(g) \cap \operatorname{supp}(h)$. That is, $G$ is a $d$-group.

Let $G$ be a group of permutations of $X$. If $G$ is a $d$-group then we shall equip $G$ with the Boolean orthogonality $\perp$ defined by $g \perp h$ if and only if $\operatorname{supp}(g) \cap$ $\operatorname{supp}(h)=\varnothing$. We let $\Sigma_{G}$ denote the topology on $X$ having the sets $\operatorname{supp}(g)$, $g \in G$, as sub-basic open sets. The condition that $G$ be a $d$-group is almost the condition that the sets $\operatorname{supp}(g), g \in G$, are basic open sets for the $\Sigma_{G}$-topology. We give a name to this latter phenomenon: $G$ is a $d^{*}$-group if the $\operatorname{sets} \operatorname{supp}(g)$, $g \in G$, form a base for the $\Sigma_{G}$-topology on $X$. Thus, $G$ is a $d^{*}$-group if and only if

(1) for all $g, h \in G$, if $x \in \operatorname{supp}(g) \cap \operatorname{supp}(h)$ then there is a $k \in G$ with $x \in \operatorname{supp}(k) \subseteq \operatorname{supp}(g) \cap \operatorname{supp}(h)$,

(2) no point of $x$ is fixed by all $g \in G$.

Lemma 3. Let $G$ be a $d^{*}$-group on the set $X$. For all $g \in G, g^{\perp \perp}=\{k \in G$ : $\operatorname{supp}(k) \subseteq \operatorname{supp}(g)\}$, where $\bar{S}$ denotes the closure of $S \subseteq X$ for the $\Sigma_{G^{-}}$topology.

Proof. Take $g \in G$ and suppose $\operatorname{supp}(k) \subseteq \overline{\operatorname{supp}(g)}$. If $g \perp h$ then $\operatorname{supp}(g)$ $\cap \operatorname{supp}(h)=\varnothing \operatorname{so} \operatorname{supp}(h) \cap \overline{\operatorname{supp}(g)}=\varnothing$ and therefore $\operatorname{supp}(h) \cap \operatorname{supp}(k)=$ $\varnothing$. That is, $k \in g^{\perp \perp}$.

Suppose on the other hand that $k \in g^{\perp \perp}$. If $\overline{\operatorname{supp}(g)}=X$ there is nothing to show. Suppose otherwise that $O$ is a non-empty open set contained in $X \backslash$ $\operatorname{supp}(g)$. Then $O=U\{\operatorname{supp}(h): \operatorname{supp}(h) \cap \operatorname{supp}(g)=\varnothing\}$ so $\operatorname{supp}(k) \cap O=$ $\cup\{\operatorname{supp}(k) \cap \operatorname{supp}(h): \operatorname{supp}(h) \cap \operatorname{supp}(g)=\varnothing\}=\varnothing$. That is, $\operatorname{supp}(k) \subseteq \overline{\operatorname{supp}(g)}$.

Corollary 4. Let $G$ be a $d^{*}$-group on the set $X$. Then the following are equivalent, for all $g, h \in G$ :

(1) $g \perp h$,

(2) $\operatorname{supp}(g) \cap \operatorname{supp}(h)=\varnothing$,

(3) $\overline{\operatorname{supp}(g)} \cap \overline{\operatorname{supp}(h)}$ has empty interior for the $\Sigma_{G^{-t o p o l o g y} \text {. }}$

We note that every group is a $d$-group in its right regular representation. The Boolean orthogonality defined in proposition is, however, trivial in this case in the sense that $g \perp h$ holds if and only if $g=1$ or $h=1$.

We note also that not every permutation group is a $d$-group. For instance if $G$ acts sharply doubly transitively on $X$ then $G$ is not a $d$-group. Indeed, since no permutation can have a one-element support the symmetric group on three or more letters is not a $d$-group. 


\section{Permutation representation}

Throughout this section $G$ will denote a group with a Boolean orthogonality $\perp$.

A subgroup $H$ of $G$ is a $\pi$-subgroup if $h_{1}^{\perp \perp} \vee \cdots \vee h_{n}^{\perp \perp} \subseteq H$ for each finite subset $\left\{h_{1}, \ldots, h_{n}\right\}$ of $H$.

The $\pi$-subgroups of $G$ ordered by inclusion clearly form a complete lattice.

LEMMA 5. Let $H$ be a $\pi$-subgroup of $G$ and $g$ an element of $G$. Let $\langle H \cup\{g\}\rangle$ be the smallest $\pi$-subgroup of $G$ containing $H \cup\{g\}$. Then $\langle H \cup\{g\}\rangle=$ $\cup\left\{F^{\perp \perp} \vee g^{\perp \perp}: F \subseteq G, F\right.$ finite $\}$.

Proof. If $F=\left\{h_{1}, \ldots, h_{n}\right\}$ is a finite subset of $H$ then $F^{\perp \perp}=h_{1}^{\perp \perp}$ $\vee \cdots \vee h_{n}^{\perp \perp}$ so $F^{\perp \perp} \vee g^{\perp \perp} \subseteq\langle H \cup\{g\}\rangle$. If $k_{1}, k_{2} \in G$ then $k_{1}^{\perp} \cap k_{2}^{\perp} \subseteq$ $\left(k_{1} k_{2}^{-1}\right)^{\perp}$, since sets of the form $S^{\perp}$ are subgroups of $G$, so $k_{k} k_{2}^{-1} \in k_{1}^{\perp \perp} \vee$ $k_{2}^{\perp \perp}$. Consequently if $k_{1} \in F_{1}^{\perp \perp} \vee g^{\perp \perp}$ and $k_{2} \in F_{2}^{\perp \perp} \vee g^{\perp \perp}$, where $F_{1}$ and $F_{2}$ are finite subsets of $H$, then

$$
k_{1} k_{2}^{-1} \in k_{1}^{\perp \perp} \vee k_{2}^{\perp \perp} \subseteq F_{1}^{\perp \perp} \vee F_{2}^{\perp \perp} \vee g^{\perp \perp}=\left(F_{1} \cup F_{2}\right)^{\perp \perp} \vee g^{\perp \perp} .
$$

Hence $\cup\left\{F^{\perp \perp} \vee g^{\perp \perp}: F \subseteq G, F\right.$ finite $\}$ is a subgroup of $G$, and therefore by its definition a $\pi$-subgroup, contained in $\langle H \cup\{g\}\rangle$, so equality holds.

We call the meet-irreducible elements of the lattice of $\pi$-subgroups of $G$ the prime $\pi$-subgroups of $G$. Thus, a $\pi$-subgroup $P$ of $G$ is a prime $\pi$-subgroup if and only if for all $\pi$-subgroups $H_{1}, H_{2}$ of $G, H_{1} \cap H_{2} \subseteq P$ implies $H_{1} \subseteq P$ or $H_{2} \subseteq P$. Equivalently, a $\pi$-subgroup $P$ is prime if and only if for all $g, h$ in $G, g^{\perp \perp} \cap h^{\perp \perp} \subseteq P$ implies $g \in P$ or $h \in P$.

We shall now see that when $G$ satisfies a certain finiteness condition for $\perp$, the prime $\pi$-subgroups of $G$ intersect in 1 . This fact allows us to represent such groups as $d^{*}$-groups of homeomorphisms.

Definition. The Boolean orthogonality $\perp$ on $G$ is finite if for all $g, h \in G$ there is a finite subset $F$ of $G$ with $g^{\perp \perp} \cap h^{\perp \perp}=F^{\perp \perp}$.

In the context of Boolean orthogonalities on rings this finiteness condition was introduced by Stewart (1975).

Proposition 6. Let $G$ be a group with a finite Boolean orthogonality $\perp$ and suppose $g_{0} \neq 1$ in $G$. Then there is a subgroup of $G$ maximal with respect to being $a \pi$-subgroup of $G$ not containing $g_{0}$. Any such $\pi$-subgroup is prime. 
Proof. The set of $\pi$-subgroups of $G$ is plainly inductive so if $g_{0} \neq 1$ then there is a subgroup $H$ of $G$ maximal with respect to being a $\pi$-subgroup not containing $g_{0}$. Assume that $H$ is not prime. Then there exist $g, k \notin H$ such that $g^{\perp \perp} \cap k^{\perp \perp} \subseteq H$. Since $g_{0} \in\langle H \cup\{g\}\rangle \cap\langle H \cup\{k\}\rangle$ there are finite subsets $F_{1}, F_{2}$ of $H$ such that

$$
\begin{aligned}
& g_{0} \in\left(F_{1}^{\perp \perp} \vee g^{\perp \perp}\right) \cap\left(F_{1}^{\perp \perp} \vee k^{\perp \perp}\right) \\
& \quad=\left(F_{1}^{\perp \perp} \cap F_{2}^{\perp \perp}\right) \vee\left(F_{1}^{\perp \perp} \cap k^{\perp \perp}\right) \vee\left(F_{2}^{\perp \perp} \cap g^{\perp \perp}\right) \vee\left(g^{\perp \perp} \cap k^{\perp \perp}\right) .
\end{aligned}
$$

Since $\perp$ is finite, there is a finite subset $F$ of $G$ with $F^{\perp \perp}=g^{\perp \perp} \cap k^{\perp \perp}$. Since $g^{\perp \perp} \cap k^{\perp \perp} \subseteq H$ then $F \subseteq H$ and $g_{0} \in H$-a contradiction, so $H$ is a prime $\pi$-subgroup.

COROllary 7. If $G$ is a group with a finite Boolean orthogonality $\pi$ then $\cap\{P \subseteq G: P$ is a prime $\pi$-subgroup $\}=1$.

It is easily seen from condition (3) for a Boolean orthogonality that each conjugate of a (prime) $\pi$-subgroup of $G$ is again a $\pi$-subgroup of $G$.

Let $G$ and $H$ be groups with Boolean orthogonalities, both denoted by $\perp$. A group homomorphism $f: G \rightarrow H$ is a $\perp$-homomorphism if $g \perp h$ in $G$ implies gf $\perp h f$ in $H$. A $\perp$-isomorphism $f: G \rightarrow H$ is a bijection such that both $f$ and $f^{-1}$ are $\perp$-homomorphisms.

We now let $X=\cup\{G / P: P$ is a proper prime $\pi$-subgroup of $G\}$ be the disjoint union of left cosets of proper prime $\pi$-subgroups of $G$.

If $H$ is a subgroup of the symmetric group on $X$ and also a $d$-group we shall equip $H$ with the Boolean orthogonality $\perp$ defined by $g \perp h$ if and only if $\operatorname{supp}(g) \cap \operatorname{supp}(h)=\varnothing$.

THEOREM 8. Let $G$ be a group with a finite Boolean orthogonality $\perp$. Then $G$ is $\perp$-isomorphic with a $d^{*}$-group of homeomorphisms of a topological space.

Proof. Given $g \in G$ we define $\hat{g}: X \rightarrow X$ by $(P k) \hat{g}=P k g$. Then $\hat{G}=\{\hat{g}$ : $f \in G\}$ is a subgroup of the symmetric group on $X$, and $g \rightarrow \hat{g}$ is an isomorphism onto $\hat{G}$ since the intersection of all prime $\pi$-subgroups of $G$ is 1 . We now see that $g \perp h$ in $G$ if and only if $\operatorname{supp}(\hat{g}) \cap \operatorname{supp}(\hat{h})=\varnothing$. Suppose $g \perp h$ in $G$. If $(P k) \hat{g} \neq P k$ then $k g k^{-1} \notin P$ That is, $g \in k^{-1} p k$ and so, since $k^{-1}$ is a prime $\pi$-subgroup and $g^{\perp \perp} \cap h^{\perp \perp} \subseteq k^{-1} p k$, we have $h \in k^{-1} p k$. That is, $(P k) \hat{h}=P k$ so $\operatorname{supp}(\hat{g}) \subseteq X \backslash \operatorname{supp}(\tilde{h})$.

Suppose conversely that $\operatorname{supp}(\hat{g}) \cap \operatorname{supp}(\hat{h})=\varnothing$. Let $P$ be a prime $\pi$-subgroup of $G$. Then $P g=P$ or $P h=p$ so $g^{\perp \perp} \subseteq P$ or $h^{\perp \perp} \subseteq P$. In any case, $g^{\perp \perp} \cap h^{\perp \perp} \subseteq P$. Since the prime $\pi$-subgroups of $G$ meet in 1 we have $g^{\perp \perp} \cap$ $h^{\perp \perp}=1$ and therefore $g \perp h$. 
Thus the relation $\perp$ on $\hat{G}$ defined by $\hat{g} \perp \hat{h}$ if and only if $\operatorname{supp}(g) \cap \operatorname{supp}(h)$ $=\varnothing$ is a Boolean orthogonality. Suppose that $g, h \in G$ and $P k_{0} \in \operatorname{supp}(\hat{g}) \cap$ $\operatorname{supp}(\hat{h})$. Then $g, h \in k_{0}^{-1} P k_{0}$. Take $k \in g^{\perp \perp} \cap h^{\perp \perp} \backslash k_{0}^{-1} P k_{0}$, so that $P k_{0} \in$ $\operatorname{supp}(k)$. If $P^{1} k_{1} \in \operatorname{supp}(k)$ then $k \notin k_{1}^{-1} P^{1} k_{1}$ so $g^{\perp \perp} \cap h^{\perp \perp} Z k_{1}^{-1} P^{1} k_{1}$. In this case $g, h \notin k_{1}^{-1} P^{1} k_{1}$ so $P^{1} k_{1} \in \operatorname{supp}(g) \cap \operatorname{supp}(h)$. That is, $\hat{G}$ is a $d^{*}$-group on $X$. Clearly each $\hat{g} \in \hat{G}$ is a homeomorphism of $X$ for the $d^{*}$-topology.

\section{Transitive representations and examples}

We have seen that every group $G$ with a finite Boolean orthogonality $\perp$ is $\perp$-isomorphic with a $d^{*}$-group of homeomorphisms of a topological space.

THEOREM 9. Let $G$ be a group with a finite Boolean orthogonality $\perp$. Then $G$ is $\perp$-isomorphic with a transitive $d^{*}$-group $\hat{G}$ of homeomorphisms of a topological

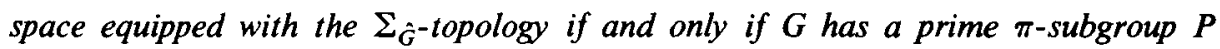
such that $\cap\left\{k^{-1} P k: k \in G\right\}=1$.

Proof. Suppose that $G$ has a prime $\pi$-subgroup $P$ whose conjugates meet in 1 . Then by proceeding as in Theorem 8 with the set of all prime $\pi$-subgroups of $G$ replaced by conjugates of $P$ we see that $G$ has a transitive representation as a $d^{*}$-group of permutations on $X=\cup\left\{G / k^{-1} P k: k \in G\right\}$.

Suppose conversely that $G$ is ( $\perp$-isomorphic with) a $d^{*}$-group of permutations acting transitively on $X$. Take $x \in X$. We see that the point stabilizer $G_{x}=$ $\{g \in G: x g=x\}$ contains a prime $\pi$-subgroup of $G$. The set of $\pi$-subgroups of $G$ contained in $G_{x}$ is inductive so let $H$ be maximal with respect to being a $\pi$-subgroup of $G$ contained in $G_{x}$. Suppose that $g, h \in H$ but $g^{\perp \perp} \cap h^{\perp \perp} \subseteq H$. Then $\langle H \cup\{g\}\rangle$ contains some $g^{\prime}$ with $x g^{\prime} \neq x$ and $\langle H \cup\{h\}\rangle$ contains some $h^{\prime}$ with $x h^{\prime} \neq x$. Then $g^{\prime} \in F_{1}^{\perp \perp} \vee g^{\perp \perp}$ and $h^{\prime} \in F_{2}^{\perp \perp} \vee h^{\perp \perp}$, for some finite subsets $F_{1}, F_{2}$ of $H$, so $\left(g^{\prime}\right)^{\perp \perp} \cap\left(h^{\prime}\right)^{\perp \perp} \subseteq H$. Then there is a $k \in G$ with $x \in \operatorname{supp}(k) \subseteq \operatorname{supp}(g) \cap \operatorname{supp}(h)$ so $k \in\left(g^{\prime}\right)^{\perp \perp} \cap\left(h^{\prime}\right)^{\perp \perp} \subseteq H \subseteq G_{x}-\mathrm{a}$ contradiction.

Thus, $H$ is a prime $\pi$-subgroup of $G$ and $\cap\left\{k^{-1} H k: k \in G\right\} \subseteq \cap\left\{k^{-1} H k\right.$ : $k \in G\} \subseteq \cap\left\{k^{-1} G_{x} k: k \in G\right\}=1$.

The motivating class of groups for the study of (finite) Boolean orthogonalities is the class of lattice-ordered groups: a lattice ordered group $G$ has a finite Boolean orthogonality $\perp$ defined by $g \perp h$ in $G$ if and only if $|g| \wedge|h|=1$ (where $|g|=g \vee g^{-1}$ ).

Our representation theorem for groups with finite Boolean orthogonalities was modelled on Holland's (1963) representation of lattice-ordered groups as groups of order-preserving permutations of chains. 
Another class of examples is provided by the (not necessarily commutative) reduced rings $R$ where we define $g \perp h$ if and only if $g h=0$. Then $g \perp$ is just the left annihilator of $g$ in $R$ which, since $R$ is reduced, is a two-sided ideal. It follows then that $g^{\perp} \subseteq(g h)^{\perp}$ for all $g, h \in R$, so $\pi$-subgroups of $R$ are in fact two-sided ideals of $R$. Our representation theorem can then be converted to a sheaf-theoretic representation for $R$. This has been carried out by Davis (1971b) and Stewart (1975).

Another-by Theorem 8 fairly typical-example is $\Gamma\left(\mathbf{R}^{2}\right)$, the homeomorphism group of $\mathbf{R}^{2}$. Indeed, suppose $g, h \in \Gamma\left(\mathbf{R}^{2}\right)$ and $x \in \operatorname{supp}(g) \cap \operatorname{supp}(h)=U$. Find inside $U$ an isometric copy $C$ of the unit open disc $O$, with $x \in C$. Let $\varphi$ : $O \rightarrow C$ be a homeomorphism from $O$ onto $C$. Define a map $\psi: \bar{O} \rightarrow \bar{O}$ as follows:

$z \psi=e^{2 \pi \mathrm{id}(z, \partial O) z}$, where $d$ is the Euclidean metric and $\partial O$ is the boundary of $O$. Now define $k: \mathbf{R}^{2} \rightarrow \mathbf{R}^{2}$ by

$$
z k= \begin{cases}z & \text { if } z \notin C, \\ z \phi^{-1} \psi \varphi & \text { if } z \in C .\end{cases}
$$

Then $k$ is a homeomorphism of $\mathbf{R}^{2}$ and $x \in \operatorname{supp}(k) \subseteq U$, so $\Gamma\left(\mathbf{R}^{2}\right)$ is a $d^{*}$-group and $\perp$, defined by $g \perp h$ if $\operatorname{supp}(g) \cap \operatorname{supp}(h)=\varnothing$, is a Boolean orthogonality.

\section{References}

W. H. Cornish (1975), 'Boolean orthogonalities and minimal prime subgroups', Comm. Algebra 3, $859-900$.

W. H. Cornish and P. N. Stewart (1977), 'Direct and subdirect decompositions of universal algebras with a Boolean orthogonality', preprint.

G. E. Davis (1971a), Orthogonality relations on groups and rings (Ph. D. thesis, Monash University, Vic.).

G E. Davis (1971b), 'Rings with orthogonality relations', Bull. Austral. Math. Soc. 4, 163-178.

G. E. Davis (1975), 'Orthogonality relations on abelian groups' J. Austral. Math. Soc. 19, 173-179.

C. Holland (1963), 'The lattice-ordered group of automorphisms of an ordered set', Michigan Math. J. 10, 399-408.

P. N Stewart (1975), 'A sheaf-theoretic representation of rings with a Boolean orthogonality', Pacific J. Math. 58, 249-254.

Department of Mathematics

La Trobe University

Bundoora, Victoria, 3083

Australia 